\title{
BMJ Open Association between social health insurance and choice of hospitals among internal migrants in China: a national cross-sectional study
}

\author{
Haiqin Wang, ${ }^{1}$ Donglan Zhang, ${ }^{2}$ Zhiying Hou, ${ }^{3}$ Fei Yan, ${ }^{1}$ Zhiyuan $\mathrm{Hou}^{1}$
}

To cite: Wang H, Zhang D, Hou Z, et al. Association between social health insurance and choice of hospitals among internal migrants in China: a national crosssectional study. BMJ Open 2018;8:e018440. doi:10.1136/ bmjopen-2017-018440

- Prepublication history and additional material for this paper are available online. To view these files, please visit the journal online (http://dx.doi org/10.1136/bmjopen-2017018440).

Received 28 June 2017 Revised 22 November 2017 Accepted 10 January 2018
Check for updates

${ }^{1}$ Department of Social Medicine, School of Public Health, National Key Laboratory of Health Technology Assessment (National Health and Family Planning Commission), Collaborative Innovation Center of Social Risks Governance in Health, Fudan University, Shanghai, China

${ }^{2}$ Department of Health Policy and Management, College of Public Health, University of Georgia, Athens, Georgia, USA ${ }^{3}$ Shanghai Health Development Research Center, Shanghai, China

Correspondence to

Dr Zhiyuan Hou;

zyhou@fudan.edu.cn

\section{ABSTRACT}

Objectives There is a tendency to pursue higher-level hospitalisation services in China, especially for internal migrants. This study aims to investigate the choices of hospitalisation services among internal migrants, and evaluate the association between social health insurance and hospitalisation choices.

Methods Data were from a 2014 nationally representative cross-sectional sample of internal migrants aged 15-59 years in China. Descriptive analyses were used to perform the distribution of healthcare facility levels for hospitalisation services, and multinomial logistic regression was applied to examine the association between social health insurance and hospitalisation choices.

Results Of the 6121 inpatient care users, only $11.50 \%$ chose the primary healthcare facilities for hospitalisation services, $44.91 \%$ chose the secondary hospitals and $43.59 \%$ preferred the tertiary hospitals. The choices presented large regional variations across the country. Compared with the uninsured, social health insurance had no statistically significant effect on patient choices of healthcare facility levels among internal migrants in China, whereas socioeconomic status was positively associated with the choices.

Conclusions Social health insurance had little influence on the hospital choice among the internal migrants. Thus, social health insurance should be consolidated and portable to enhance the proper incentive of health insurance on healthcare seeking behaviours.

\section{INTRODUCTION}

The health status and healthcare utilisation among internal migrants, a population defined as those who live in a new residence for more than 1 month but do not have a local 'Hukou' of the new residence (registered resident certificate), has become an important research focus in China. ${ }^{1-5}$ The reason for this special interest is that within China's three-tiered healthcare delivery system, there has been a tendency for people to pursue higher-level hospitalisation service, with admissions to tertiary hospitals increasing by 13.5 percentage points
Strengths and limitations of this study

- We aim to investigate the association between social health insurance and hospital choice among internal migrants in China.

- Data are from the 2014 nationally representative cross-sectional sample of internal migrants in China, and multinomial logistic regressions are used.

- The sensitivity analyses are conducted on total sample and two subsamples to get robust results.

- It cannot be interpreted as causal relationship due to the cross-sectional study.

(from $22.5 \%$ to $36.0 \%$ ) and admissions to primary care facilities decreasing from $38.4 \%$ to $26.4 \%$ between 2009 and $2015{ }^{6}$ This tendency was more marked in internal migrants; $43.6 \%$ of migrants with admission chose the tertiary hospitals in 2014, higher by 7.6 percentage points than that in general population. ${ }^{7}$ However, with this tendency, the delivery system has been becoming more fragmented and hospital-centred, ${ }^{8}$ and thus leads to dramatic escalation of healthcare expenditures and jeopardises the Chinese goal of providing affordable and equitable access to quality healthcare for all citizens.

The Chinese government is currently attempting to use social health insurance policy levers to reverse this trend. To guide healthcare seeking behaviour, the social health insurance introduced a differential reimbursement policy tailored to the level of healthcare facilities, which ruled that the higher the facility level, the less the insurance reimbursement rates. ${ }^{9} 10$ In theory, there are two primary ways that health insurance affects healthcare seeking behaviour: the income effect and substitution effect. ${ }^{11}{ }^{12}$ On the one hand, the income effect indicates that the insured face a lower price of health services than the uninsured, and will increase the utilisation of health services, especially at 
higher-level hospitals. ${ }^{11} 12$ On the other hand, different levels of healthcare facilities are substitutes to some degrees, and an increase in the price of health services at one level of facilities would cause patient to consume more health services at the other levels, namely the substitution effect. ${ }^{11}$ The differential reimbursement rates of health insurance change the relative prices of health services at different facility levels, and would increase the likelihood of using lower-level healthcare facilities. Considering these two influencing ways together, health insurance may have the mixed effects on healthcare seeking behaviour. However, to date there is no empirical evidence on the role of health insurance in healthcare seeking behaviours for internal migrants.

In addition, the choice of healthcare providers can be determined by patient characteristics, health insurance and provider characteristics. ${ }^{13}{ }^{14}$ Healthcare providers differ according to the price and quality of care. When making choices of providers, patients generally seek for high-quality care while minimising cost, and choose the provider that best fits their preferences and needs. ${ }^{13}$ Health insurance could reduce the price of health services paid by patients. However, empirical evidence is mixed on how patients choose different levels of providers for treatment. Studies in Nepal and India found that the price of health services and household income had stronger effects on hospital choice than the hospital quality or severity of illness, ${ }^{1516}$ whereas studies from Dutch hospitals found the positive influence of care quality on choice. ${ }^{17}$ In China, most of the literature focused on whether patients used health services, but only a few studies investigated the choices of patients conditional on treatment. A study in rural China found that the differential health insurance policies influenced the choice of hospital, and in particular, as the reimbursement ceiling rose, patients increasingly chose higher-level hospitals. ${ }^{18}$ Another research confirmed the impact of the differential health insurance reimbursement policy by the facility level among rural patients with diabetes. ${ }^{14}$ They found that increase of outpatient reimbursement rates at township or county hospitals significantly increased the outpatient service utilisation at these facilities, although no such relationship for village clinics. Evidence from the China National Health Services Survey in 2008 presented that patients chose primary care facilities mainly due to distance and price of care, but chose hospitals because they attached more importance on perceived quality of care. $^{19}$

In China, the social health insurance system has been fragmented into three separate insurance schemes: the New Rural Cooperative Medical Scheme (NCMS), the Urban Resident Basic Medical Insurance (URBMI) and the Urban Employee Basic Medical Insurance (UEBMI). These three health insurance schemes covered about $95 \%$ of the total population in China, ${ }^{20}$ and they are designed to target different populations and are difficult to transfer between home city and a city of new residence. The NCMS targets the registered rural population; the
URBMI and UEBMI target the urban non-employee residents and employees, respectively. ${ }^{21}$ However, they are pooled and administrated at the county or prefecture level. Each county or prefecture designs its own benefit package and reimbursement policy of health insurance, leading to variation and non-portability across geographic areas. Internal migrants are usually covered by the health insurance in their hometown, which mainly insures healthcare provided in that location. ${ }^{22}$ After moving from their hometown to other cities, migrants tend to use health services at their new city of residence. Therefore, the health services they use at their new residence are generally out of the coverage network of their health insurance, thereby ineligible for the reimbursement of health insurance. ${ }^{23}$ To achieve universal health coverage, the Chinese government recently issued policies to allow migrants to participate in URBMI or UEBMI according to their employment status, and to guide the transfer of health insurance from hometowns to the living prefectures. ${ }^{24}$ However, it is a prefecture-based policy, and each prefecture can decide the implementation of its own health insurance transferability policy. Migrants' accessibility to local health insurance may vary by prefecture. Some areas have also tried to consolidate the NCMS and URBMI into one unified health insurance scheme. ${ }^{25}$ Therefore, it is necessary to evaluate the influence of health insurance in healthcare seeking behaviours for internal migrants.

Using the 2014 wave of China's annual National Internal Migrant Dynamic Monitoring Survey, the present study examined hospitalisation choices and their determinants among internal migrants, and especially focused on the effect of social health insurance on hospitalisation choices. The study has two objectives: (1) to investigate nationally the choices of the levels of healthcare facilities for hospitalisation services among internal migrants, and the regional variation of these choices and (2) to evaluate the association between social health insurance and hospitalisation choices. The study will help in developing interventions that can change the current fragmented and hospital-centric system. This paper is among the first to investigate the association between social health insurance and the choices of the levels of healthcare facilities for hospitalisation services among internal migrants in China.

\section{METHODS}

\section{Data and study design}

Data used were from the 2014 wave of China's annual National Internal Migrant Dynamic Monitoring Survey. ${ }^{26}$ This survey was conducted by the National Health and Family Planning Commission of China in May 2014. This is a national cross-sectional survey representing internal migrants aged 15-59 years, and aims to examine the socioeconomic status of internal migrants, determinations of healthcare services and health outcomes. In this survey, internal migrants are defined as those who do not 
have the 'Hukou' of local counties/districts and have been living in locals for $>1$ month, including migrants from both rural and urban areas.

The survey was drawn using the stratified multistage random sampling method by probability proportional to size (PPS), and the annual national data on internal migrants from each province in 2013 was considered as the basic sampling frame. The survey covered 348 cities from all 32 provincial units in China. Within each city, townships were randomly selected and followed by village or neighbourhoods by PPS. In each village or neighbourhood, 20 internal migrants were randomly selected to participate in the survey, finally reaching a total sample size of 200937 respondents. All respondents were interviewed face-to-face by trained interviewers, using a structured questionnaire. Approvals were obtained from the institutional review board, and the participants provided consent for the same.

Questionnaires included demographic information and family structures, socioeconomic status, migration characteristics, health insurance, healthcare services and family planning services. In this paper, we focused on the choice of different levels of healthcare facilities for hospitalisation services (inpatient care). Thus, migrants who used hospitalisation services in the previous year of the survey were included in our analysis, and the sample size was 6121 inpatient services users.

\section{Research hypothesis and measurements}

Our hypothesis was that social health insurance would increase the likelihood of using lower-level healthcare facilities. The dependent variable we focused on was patient choice of healthcare facilities for hospitalisation services. In China, there are three levels of healthcare facilities that patients can choose to be admitted to: the primary care facility, the secondary hospital and the tertiary hospital. Our primary predictor of interest was social health insurance status, which was categorised into four subgroups: no social health insurance, coverage by one of the three types of social health insurance-NCMS, URBMI or UEBMI. Social health insurance sets higher reimbursement rates for lower-level healthcare facilities, for example, in 2014, the reimbursement rates from the NCMS were around $80 \%, 70 \%$ and $55 \%$ for inpatient services at the primary care facilities, the secondary and tertiary hospitals, respectively. ${ }^{27}$

Controlled variables included demographic characteristics, socioeconomic status, migration characteristics and hospitalisation information. Demographic characteristics included gender, age and marital status. Socioeconomic status was measured by 'Hukou' status, education, income and whether the respondent had a job. Hukou is the record in the residency registration system in China; people can be registered as having either a rural or urban Hukou, at the time of birth. Monthly income per capita was ranked and divided into five quintiles (the lowest, lower, middle, higher and the highest levels), with the lowest level as the reference group. Migration characteristics were measured by living areas, migration region and time. Migrants may move and live in urban or suburban areas. Migration region was categorised into three types: migration across provinces; migration across cities but within a province and migration across counties but within a city. Migration time was categorised into four types: 1year and below; 2-3years; 4-6years and 7years and above. Finally, hospitalisation information included the place and reason for hospitalisation. The places of hospitalisation can be a city of new residence, hometown or other cites. The reasons for hospitalisation included diseases, childbirth and others.

\section{Statistical analysis}

We first described the distribution of healthcare facility levels for hospitalisation services among internal migrants, and its regional variation by province. Descriptive analyses were then performed to compare the sample characteristics by the level of healthcare facility.

Second, multinomial logistic regressions were employed to examine the association between social health insurance status and the choice of healthcare facility levels. The secondary hospital was taken as the reference group, because China would like to make most inpatient services to be used in the county, and the county (secondary) hospitals are the main body for inpatient services and accounted for the largest proportion of all inpatient services. All regression models adjusted for respondents' demographic characteristics (gender, age and marital status), socioeconomic status (rural or urban 'Hukou', education, income, having a job or not), migration characteristics (moving to urban or suburban areas, migration region and time), hospitalisation information (place and reason) and having commercial health insurance or not. City fix effects were also controlled for in the models. To address the unbalance in general characteristics between the insured and uninsured groups, we further added multinomial logistic regressions with propensity-score weighting to confirm the association between social health insurance and choice of hospitals. In addition, for the sensitivity analyses, all regressions were run on both total sample and two subsamples of disease and childbirth to get robust results.

To further explore the relationships observed in the individual choice model and explain the reasons behind the patient choice from the social health insurance policy perspective, we used bivariate analyses to compare the health insurance reimbursement probability and reimbursement ratio among those who received reimbursement by the level of healthcare facilities, which were reported by patients after they used the hospitalisation services.

All of the analyses were conducted for the total sample, and separately for the disease and childbirth subsamples. ORs and 95\% CIs were presented. All statistical analyses were performed using Stata V.12.0 (StataCorp, College Station, Texas, USA). 


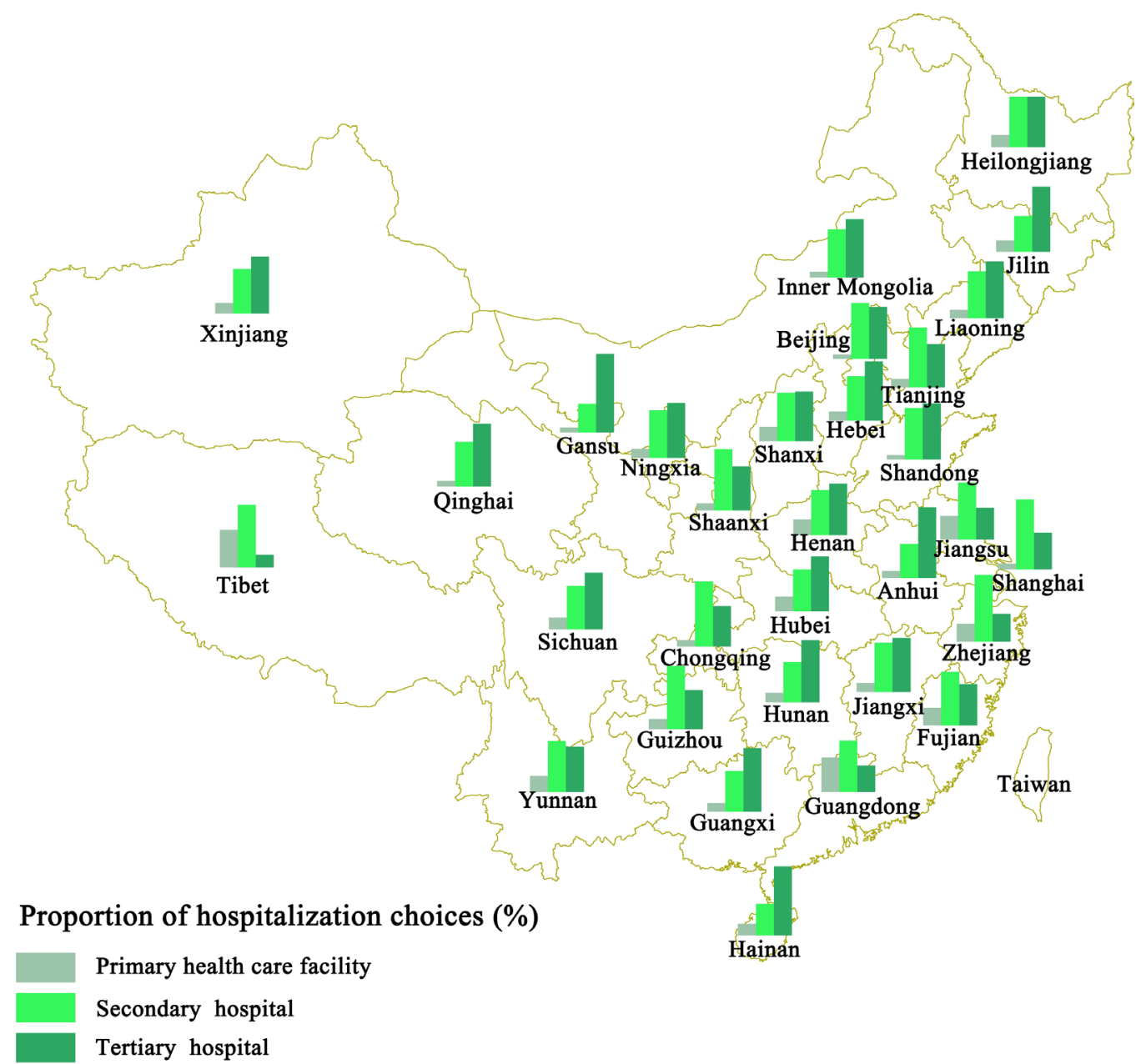

Figure 1 Choices of healthcare facility Levels for hospitalisation services among internal migrants by province, 2014.

\section{RESULTS}

Choices of healthcare facility levels for hospitalisation services

Of the 6121 respondents, only $11.50 \%$ chose the primary healthcare facilities for hospitalisation services, $44.91 \%$ chose the secondary hospitals and $43.59 \%$ preferred the tertiary hospitals. Figure 1 presents the regional variation on the choice of healthcare facility levels for hospitalisation services among internal migrants in China. As can be seen, in the more economically developed municipalities (Beijing, Shanghai, Chongqing and Tianjin) and eastern coastal regions (Shandong, Jiangsu, Zhejiang, Fujian and Guangdong provinces), migrants were more likely to choose secondary hospitals, whereas in the less developed regions (Central, Western and Northeastern China), the most common choice was tertiary hospitals.

\section{Characteristics of the sampling respondents}

Table 1 provides the sample characteristics totally and by healthcare facility levels. Overall, $58.08 \%$ of the respondents were covered by the NCMS, $7.09 \%$ and $20.96 \%$ were covered by the URBMI and UEBMI, respectively, while $13.87 \%$ had no social health insurance. About $5.85 \%$ of the respondents purchased commercial health insurance.
Respondents with admission to primary care facilities were more likely to be uninsured or with NCMS coverage, whereas respondents with admission to the secondary or tertiary hospitals were more likely to be covered by the URBMI or UEBMI.

Three-fourths of the respondents were female, and were on average aged 32 years; $82.50 \%$ were registered as having a rural 'Hukou', and nearly $60 \%$ received an education of junior high school or below; $70.79 \%$ of respondents moved to urban areas, half of the respondents migrated across provinces and $42 \%$ have lived in the new residence for more than 4 years; $70.33 \%$ chose to receive hospitalisation services at their new city of residence, and more than half of all hospitalisation services were for childbirth.

\section{Factors associated with choices of healthcare facility levels}

Table 2 presents the results from the multinomial logistic regression models that examined the association between social health insurance coverage and the choice of healthcare facility levels. In the total sample, compared with the uninsured, having any type of social health insurance had no significant relationship with the choice between secondary and tertiary hospitals. Coverage by the NCMS and URBMI had no significant influence on the choice 
Table 1 Sample characteristics by levels of healthcare facilities, 2014 (\%)

\begin{tabular}{|c|c|c|c|c|}
\hline \multirow[b]{2}{*}{ Characteristics } & \multirow{2}{*}{$\begin{array}{l}\text { Total } \\
\mathrm{n}=6121\end{array}$} & \multicolumn{3}{|c|}{ Levels of healthcare facilities } \\
\hline & & Primary $n=704$ & $\begin{array}{l}\text { Secondary } \\
n=2749\end{array}$ & Tertiary $n=2668$ \\
\hline Social health insurance & & & & 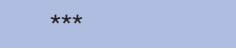 \\
\hline No insurance & 13.87 & 16.90 & 13.75 & 13.19 \\
\hline NCMS & 58.08 & 66.48 & 59.88 & 54.01 \\
\hline URBMI & 7.09 & 6.53 & 6.40 & 7.95 \\
\hline UEBMI & 20.96 & 10.09 & 19.97 & 24.85 \\
\hline Commercial health insurance & 5.85 & 4.12 & 5.67 & 6.48 \\
\hline Female & 76.78 & 73.72 & 78.06 & $76.27^{\star}$ \\
\hline Age (years) $\dagger$ & $31.95(8.51)$ & $31.90(8.72)$ & $31.17(8.25)$ & $32.78^{\star \star \star}(8.65)$ \\
\hline Married & 92.53 & 91.05 & 93.31 & 92.13 \\
\hline Rural Hukou & 82.50 & 93.04 & 85.41 & $76.72^{\star \star \star}$ \\
\hline Education & & & & 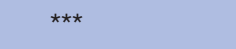 \\
\hline Primary school or below & 13.04 & 18.61 & 13.13 & 11.47 \\
\hline Junior high school & 45.76 & 54.97 & 48.34 & 40.67 \\
\hline Senior high school & 20.54 & 17.76 & 19.61 & 22.23 \\
\hline College or above & 20.67 & 8.66 & 18.92 & 25.64 \\
\hline Monthly income per capita (1000 RMB) $\dagger$ & $2.28(2.42)$ & $1.97(1.39)$ & $2.25(2.42)$ & $2.39^{\star \star \star}(2.61)$ \\
\hline Having any job & 56.49 & 59.38 & 56.49 & 55.73 \\
\hline Moving to urban area & 70.79 & 54.55 & 67.37 & $78.60^{\star \star \star}$ \\
\hline Migration region & & & & *** \\
\hline Across province & 48.93 & 58.52 & 53.91 & 41.27 \\
\hline Across city within province & 30.94 & 28.13 & 26.96 & 35.79 \\
\hline Across county within city & 20.13 & 13.35 & 19.13 & 22.94 \\
\hline Migration time (years) & & & & *** \\
\hline $0-1$ & 34.83 & 38.78 & 38.45 & 30.06 \\
\hline $2-3$ & 23.07 & 21.73 & 21.94 & 24.59 \\
\hline $4-6$ & 20.52 & 20.17 & 19.68 & 21.48 \\
\hline 7 or more & 21.58 & 19.32 & 19.93 & 23.88 \\
\hline Places of hospitalisation & & & & $\star * \star *$ \\
\hline New city of residence & 70.33 & 63.92 & 64.35 & 78.19 \\
\hline Hometown & 21.74 & 32.39 & 29.90 & 10.53 \\
\hline Others & 7.92 & 3.69 & 5.75 & 11.28 \\
\hline Reasons of hospitalisation & & & & $* \star \star$ \\
\hline Disease & 29.21 & 33.52 & 25.32 & 32.08 \\
\hline Childbirth & 58.90 & 55.40 & 62.93 & 55.66 \\
\hline Others & 11.89 & 11.08 & 11.75 & 12.26 \\
\hline
\end{tabular}

${ }^{*} \mathrm{P}<0.05,{ }^{* *} \mathrm{P}<0.01,{ }^{* * *} \mathrm{P}<0.001$.

†Mean, SD.

NCMS, New Rural Cooperative Medical Scheme; UEBMI, Urban Employee Basic Medical Insurance; URBMI, Urban Resident Basic Medical Insurance.

between primary care facilities and secondary hospitals either, although the UEMBI significantly decreased the probability of choosing primary care facilities (OR 0.47, $95 \%$ CI 0.33 to 0.67$)$. Having commercial health insurance had no association with the choice of healthcare facility levels. These results on the relationships between health insurance and the choices of hospitalisation services still held for the disease and childbirth subsamples.

Respondents with better socioeconomic status were more likely to choose higher level of healthcare facilities. In comparison with secondary hospitals, registration status of rural 'Hukou' significantly reduced the 


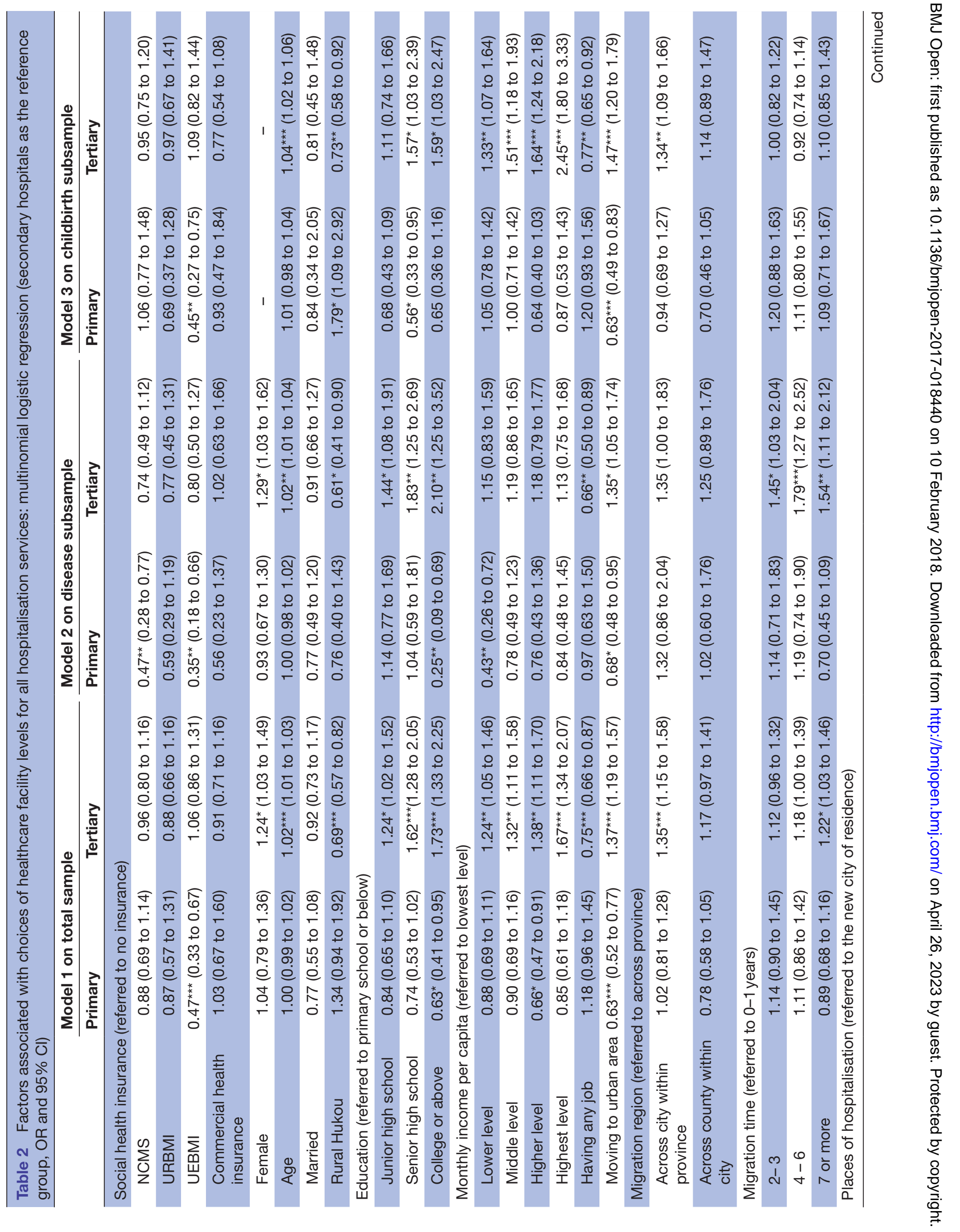


likelihood of using tertiary hospitals by $31 \%$. Both better education and income increased the probability of choosing tertiary hospitals, but decreased the probability of choosing primary care facilities. However, respondents with any job were less likely to use the tertiary hospitalisation services than those with no job.

With regard to the migration characteristics, moving to urban areas significantly increased the likelihood of using tertiary hospitals (OR 1.37, 95\% CI 1.19 to 1.57), but significantly decreased the likelihood of using primary care facilities (OR $0.63,95 \%$ CI 0.52 to 0.77 ). Compared with migration across provinces, those who migrated across cities but within a province were more likely to select tertiary hospitals, which was mainly embodied in the childbirth subsample. The longer the migration time, the higher the likelihood for respondents to opt for the tertiary hospitals, which was mainly embodied in the disease subsample.

In addition, female and older people were more likely to opt for tertiary hospitals. Compared with hospitalisation at their new city of residence, respondents who were hospitalised at their hometown were significantly less likely to choose tertiary hospitals (OR $0.29,95 \%$ CI 0.25 to 0.34 ), whereas hospitalisation at other places tended to be admitted at higher-level hospitals.

We also conducted a confirmatory analysis to address the unbalance in general characteristics between the insured and uninsured groups. We compared the propensity-score-weighted frequency distributions for the sample characteristics between the insured and uninsured groups, and after propensity-score weighting, the differences between the two groups became insignificant. The results from multinomial logistic regressions after the propensity-score weighting were very similar to the results without propensity-score weighting (see online supplementary appendix table 1). Therefore, we confirmed that the unbalance in general characteristics would not affect our analysis results.

In addition, there might be difference in the effect of social health insurance schemes on the hospitalisation choices between the new cities of residence and at their hometown. To examine whether there is difference or not, we further conducted the multinomial logistic regressions by places of hospitalisation (table 3 ). The relationship did not change, showing that social health insurance coverage was insignificantly associated with hospitalisation choices for hospitalisation subsamples either at the new city of residence or hometown.

\section{Exploration of the reasons behind patient choices from the health insurance reimbursement policy}

Figure 2 provides the comparison of reimbursement probability and reimbursement ratio among those who received reimbursement from social health insurance across the level of healthcare facilities. Of the total sample, 3615 respondents $(68.57 \%)$ received reimbursements through social health insurance for their hospitalisation services. The higher the level of the healthcare facility, 


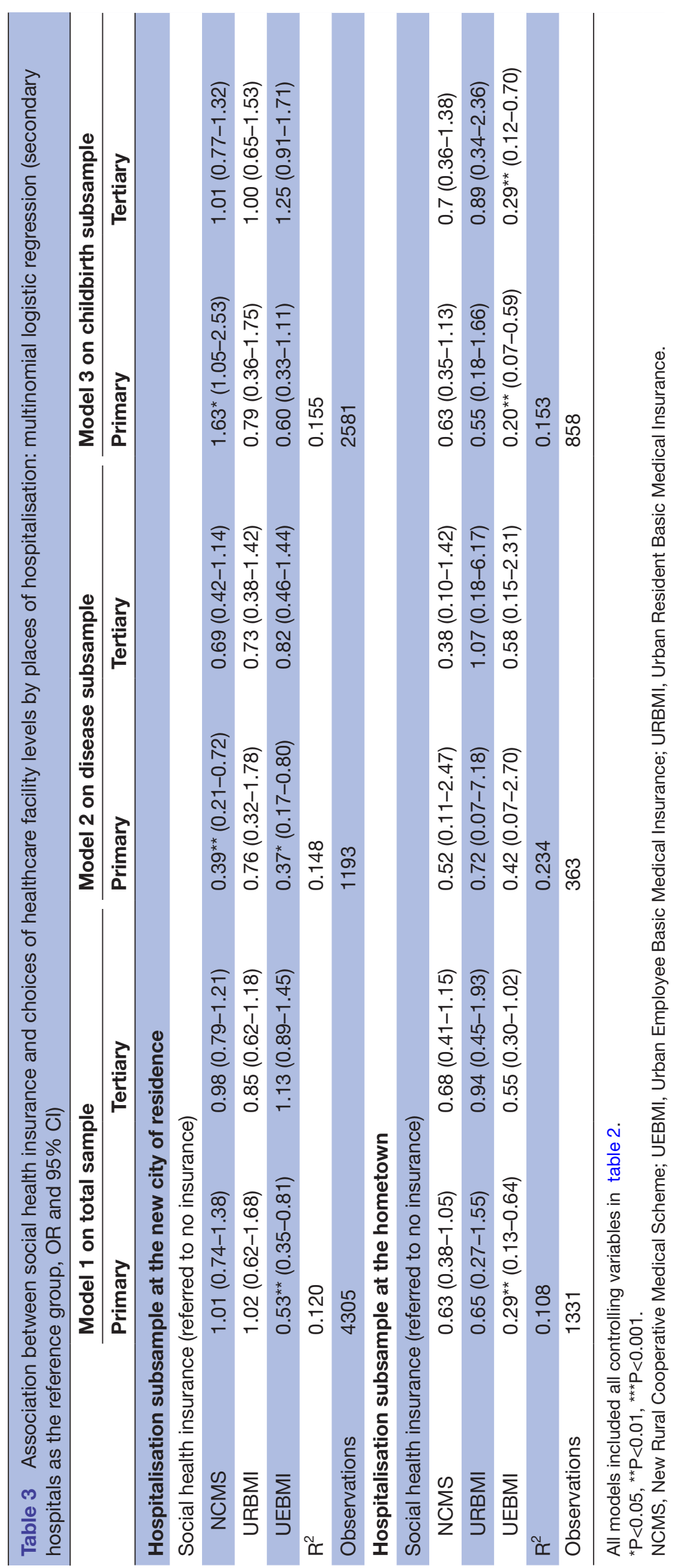


A

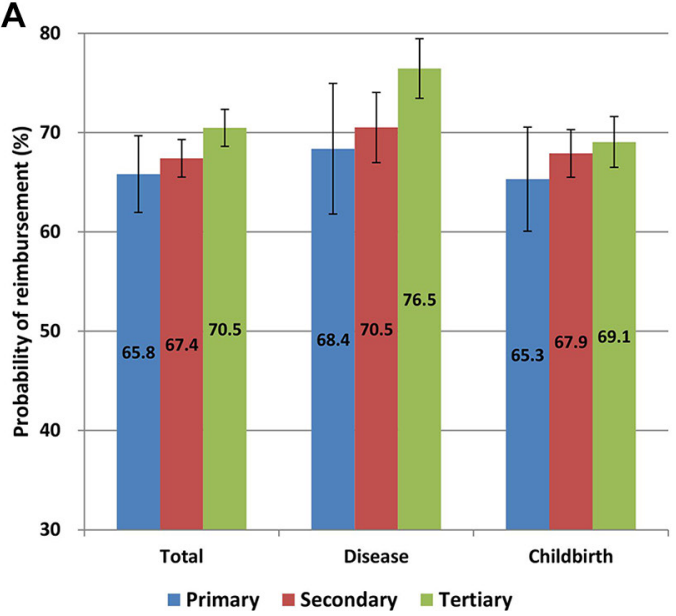

B

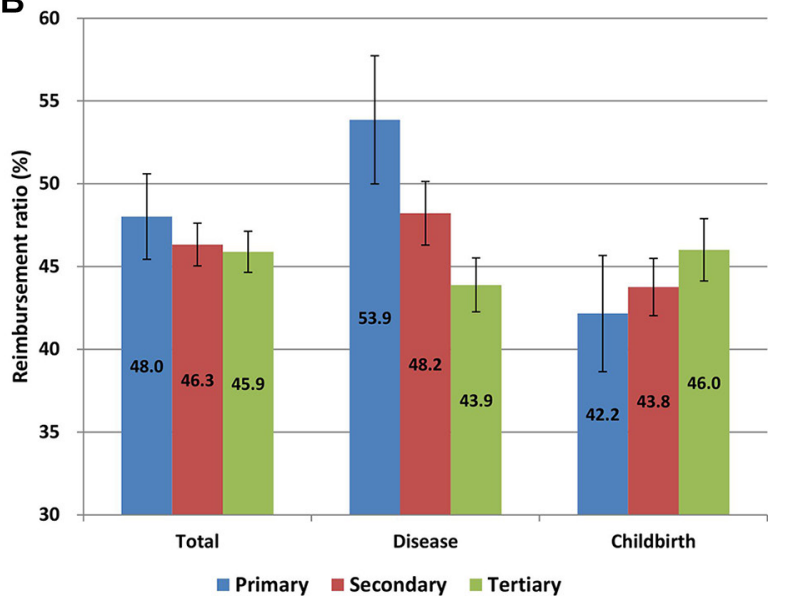

Figure 2 Health insurance reimbursement by levels of healthcare facilities: (A) reimbursement probability; (B) reimbursement ratio among those who received reimbursement. Data are presented as percentages $(95 \% \mathrm{Cl})$.

the larger the probability that respondents received reimbursement from social health insurance. Once they got reimbursement, however, the higher the facility level, the lower the reimbursement ratio. The reimbursement difference by facility level mainly occurred for disease subsample rather than for childbirth subsample. Among the disease subsample, the probability of getting reimbursement was $6-8$ percentage points higher for tertiary hospitals than for the other two lower-level facilities $(76.5 \%$ vs $70.5 \%$ or $68.4 \%)$, but the reimbursement ratio among those who received reimbursement was 5-10 percentage points lower for tertiary hospitals than for the other two lower-level facilities $(43.9 \%$ vs $48.2 \%$ or $53.9 \%)$.

\section{DISCUSSION}

This study was the first study that investigated the hospitalisation choices among internal migrants in China, and the association between social health insurance and these choices. It showed that the primary care facilities, secondary and tertiary hospitals accounted for $11.50 \%$, $44.91 \%$ and $43.59 \%$ of hospitalisation services among internal migrants, respectively, and this distribution presented large regional variations across the country. This study also found that social health insurance coverage had no statistically significant impact on patient choices of healthcare facility levels among internal migrants in China, whereas socioeconomic status was positively associated with the choices.

In principal, social health insurance policy sets higher reimbursement rates for lower-level healthcare facilities in China, which assumed to guide patients to choose the lower-level facilities. Previous studies concluded that the NCMS could affect healthcare seeking behaviour among residents. ${ }^{23}{ }^{28}$ However, it was not the case among internal migrants. Compared with primary care facilities, services at tertiary hospitals have much higher cost. Overutilisation of higher-level hospitals would lead to cost escalation. But social health insurance failed to play an important role in guiding internal migrants' healthcare seeking behaviours. First, the three social insurance schemes in China are pooled and administered at county or city levels, and they are not portable across regions. ${ }^{29}$ Generally, residents are enrolled in a health insurance policy and use healthcare at their hometown, whereas internal migrants are enrolled in their hometown insurance policy but use healthcare in their new cities of residence. For internal migrants, healthcare in their new cities of residence is generally out of the coverage network of their hometown health insurance, and they are ineligible for the insurance reimbursement. The separation between the location of the health insurance and that of the healthcare itself makes internal migrants less likely to be affected by health insurance policy, and therefore puts them in a similar situation to respondents without health insurance. Second, tertiary hospitals are more likely to be covered by internal migrants' hometown health insurance than primary care facilities. Our data showed that the higher the level of the healthcare facility where the internal migrants received inpatient care, the larger the probability that they obtained reimbursement from social health insurance. Third, the gap of reimbursement ratio among those who received reimbursement by the facility level was only $5-10$ percentage points for inpatient services due to diseases, which was too small to attract patients to choose the lower-level facilities. This was consistent with other study on choices of outpatient care providers in rural China. ${ }^{14}$ These insurance policies failed to meet the reimbursement principle of the social health insurances, ${ }^{30}$ and could be a distortion to the role of social health insurances in guiding patients. The fragmentation and non-portability of health insurance schemes across regions may limit its role in internal migrants' choices for inpatient services. Several studies have suggested that consolidating the three health insurance schemes, reducing the barriers to reimbursement for internal migrants and widening the gap of reimbursement ratio among facility levels ${ }^{3132}$ may help social health 
insurances function well in the role of guiding internal migrants to seek healthcare.

In addition, patients took into consideration cost and quality of care. Health services at different levels of facilities were only partially substituted, because there were large variations on service package and quality of care by facility levels. The previous studies documented the poor quality of care in primary care facilities, and well-trained health workers were generally concentrated in hospitals in China. ${ }^{33}$ The quality of health services at hospitals was much higher than that of primary care facilities in China. ${ }^{14}$ As shown by our data, the slight gap of reimbursement rate by facility levels cannot offset the large gap on quality and service package. The differential reimbursement policy may make functions only if the quality of care in primary care facilities would be improved and no large gap of quality occurred by facility levels. Therefore, there is a need for primary care facilities to improve their health services to attract patients.

We also found that better socioeconomic status was associated with the higher probability of admission to tertiary hospitals, which is consistent with previous studies. ${ }^{28} 3536$ However, respondents having jobs were less likely to choose tertiary hospitals in comparison with the secondary hospitals, which is not consistent with the results of most studies suggesting that those with better socioeconomic status were more likely to use expensive health services. A study on migrants in Guangzhou city found the similar results that the employed migrants had significantly lower 2-week physician consultation rate (3.4\% vs $6.8 \%)$ and annual hospitalisation rate $(4.5 \%$ vs $14.5 \%)$ than unemployed migrants. ${ }^{37}$ They also found that employed migrants tended to be healthier and may not need tertiary health services; about $75 \%$ of employed migrants and $60 \%$ of unemployed migrants rated their health status as being 'excellent or good'. There may be the 'healthy worker effect' that workers often exhibit better health status than the general population due to the exclusion of the unhealthy population from employment. ${ }^{38}$ In addition, the employed migrants had less free time than those unemployed migrants, but tertiary hospitals were generally located farther from home and had the longer waiting time than the secondary hospitals. ${ }^{37}$ And some unemployed migrants moved to the cities only to seek tertiary health services rather than to seek jobs. These reasons may explain the lower possibility to choose tertiary hospitals instead of the secondary hospitals among employed migrants.

More interestingly, there were large regional variations on hospitalisation choices. Respondents in more economically developed eastern regions were more likely to select secondary hospitals, while respondents in those less developed central and western regions mostly chose tertiary hospitals. The reasons for these choices may be the variations of the capacity of healthcare facilities and hierarchical healthcare system policy across regions. The primary care and secondary facilities were equipped with more healthcare resources and had better care quality in eastern regions than western regions. Also with constant advances in healthcare reform, eastern areas such as Beijing, Shanghai, Zhejiang, Jiangsu are taking the leading role in setting up the hierarchical healthcare system, focusing on capacity building of the primary care facilities and secondary hospitals. ${ }^{39}$ Therefore, strengthening the primary care and secondary facilities in less developed regions is necessary in order to guide patients' behaviours.

The major contribution of this study was that we sought to identify and compare different choices on hospitalisation among internal migrants holding different social health insurances. Although previous studies have observed that domestic migration affected internal migrants' health and health seeking behaviours, ${ }^{233140-43}$ research that addressed the relationship between social health insurance and hospitalisation choices among internal migrants was scarce. However, the study also had several limitations. First, it was a cross-sectional study, and, therefore, can only be used to explore associations between social health insurance and internal migrants' choices on the healthcare facilities. The relationships that we inferred cannot be interpreted as causal in nature. Second, the severity of diseases may directly affect the choices of healthcare facilities due to inpatient services, and unfortunately this survey did not measure this, which may lead to the omitted variables bias. To reduce this bias, we conducted a sensitivity analysis on two subsamples of diseases and childbirth, and found there was little variation of severity for the childbirth subsample. Third, we cannot distinguish the income effect and substitution effect of health insurance, and only capture the overall effect of health insurance on healthcare seeking behaviour.

\section{CONCLUSION}

Our study suggests that social health insurance had little influence on the hospital choice among internal migrants. Social health insurance schemes should be consolidated and portable, which would enhance the proper incentive of health insurance on healthcare seeking behaviours. The capacity building of primary care facilities and secondary hospitals are also important to increase the utilisation of these facilities.

Fortunately, some big progress has been made to achieve the portability of health insurance. New policies have been issued to make migrants eligible for health insurance locally, or to change health insurance account from their hometown to the living residence..$^{24}$ And based on the recently established national health insurance information system, healthcare at the living residence for migrants can be immediately reimbursed by health insurance at their hometown. The central government issued a national policy to consolidate the different health insurance schemes in 2016. ${ }^{44}$ These policies would improve the protection function of health insurance for migrants.

Acknowledgements The authors thank the National Health and Family Planning Commission of China for providing the data. The authors also thank Keith Dear from Duke Global Health Institute for her revision advices. 
Contributors Zhiyuan Hou contributed to the conceptualisation and design of the study. Zhiyuan Hou and HW analysed the data. HW drafted the manuscript, and Zhiyuan Hou and DZ extensively revised the manuscript, and FY and Zhiying Hou also revised the manuscript. All authors read and approved the final manuscript being submitted.

Funding This work was supported by the National Nature Science Foundation of China (grant number 71403007).

Competing interests None declared.

Patient consent Obtained.

Ethics approval National Health and Family Planning Commission of China. Provenance and peer review Not commissioned; externally peer reviewed. Data sharing statement The data used in this paper were provided by the National Health and Family Planning Commission of China. We signed a legally binding agreement with the Commission that we would not share the original data to any third parties. Although researchers who are interested can apply to get access to the data at: http://www.moh.gov.cn/ldrks/s7848s/201510/8d4c69223d4c 49ca871fdd6fdc57aba5.shtml and Email: Idrkzxsj@163.com, it is up to the National Health and Family Planning Commission to make the final decision.

Open Access This is an Open Access article distributed in accordance with the Creative Commons Attribution Non Commercial (CC BY-NC 4.0) license, which permits others to distribute, remix, adapt, build upon this work non-commercially, and license their derivative works on different terms, provided the original work is properly cited and the use is non-commercial. See: http://creativecommons.org/ licenses/by-nc/4.0/

(C) Article author(s) (or their employer(s) unless otherwise stated in the text of the article) 2018. All rights reserved. No commercial use is permitted unless otherwise expressly granted.

\section{REFERENCES}

1. Shao C, Meng X, Cui S, et al. Income-related health inequality of migrant workers in China and its decomposition: an analysis based on the 2012 China labor-force dynamics survey data. J Chin Med Assoc 2016;79:531-7.

2. Mou J, Griffiths SM, Fong $\mathrm{H}$, et al. Health of China's rural-urban migrants and their families: a review of literature from 2000 to 2012. Br Med Bull 2013;106:19-43.

3. Wei Z, Hu C, Wei X, et al. Service utilization for mental problems in a metropolitan migrant population in china. Psychiatr Serv 2013;64:645-52.

4. Chen H, Zhu Z, Sun D, et al. The physical and psychological health of migrants in Guangzhou, China. INQUIRY: The Journal of Health Care Organization, Provision, and Financing 2016;53:1-8.

5. Sun $X$, Chen M, Chan KL. A meta-analysis of the impacts of internal migration on child health outcomes in China. BMC Public Health 2015;16.

6. National Health and Family Planning Commission of China. China health statistical yearbook 2015. Beijing: Peking Union Medical College Press, 2016.

7. Wang $\mathrm{H}$, Wang $\mathrm{Y}$, Hou Z. Does health insurance impact on the hospital seeking behavior among floating population? Evidence from the 2014 National floating population dynamic monitoring survey. Chinese Journal of Health Policy 2016:61-6. In Chinese.

8. Yip W, Hsiao W. Harnessing the privatisation of China's fragmented health-care delivery. Lancet 2014;384:805-18.

9. National Health and Family Planning Commission of China. Notice on key missions of new rural cooperative medical scheme. 2014-0708 http://www.nhfpc.gov.cn/jws/s3581sg/201407/d8abe06c1a2d4c 17b78e7b14c860579a.shtml

10. Ministry of Human Resources and Social Security of China. Notice on urban residents basic medical insurance. 2015-01-27 http://www. mohrss.gov.cn/SYrlzyhshbzb/shehuibaozhang/zcwj/yiliao/201502/ t20150209 151708.html

11. Santerre RE, Neun SP. Health economics: theories, insights, and industry studies. 5th edn: Cengage Learning, 2009.

12. Nyman JA. Health insurance theory: the case of the missing welfare gain. Eur J Health Econ 2008;9:369-80.

13. Victoor A, Delnoij DM, Friele RD, et al. Determinants of patient choice of healthcare providers: a scoping review. BMC Health Serv Res 2012;12:272.

14. Zhang L, Wang Z, Qian D, et al. Effects of changes in health insurance reimbursement level on outpatient service utilization of rural diabetics: evidence from Jiangsu Province, China. BMC Health Serv Res 2014;14:185

15. Morey ER, Sharma VR, Mills A. Willingness to pay and determinants of choice for improved malaria treatment in rural Nepal. Soc Sci Med 2003;57:155-65.

16. Borah BJ. A mixed logit model of health care provider choice: analysis of NSS data for rural India. Health Econ 2006;15:915-32.

17. Dijs-Elsinga J, Otten W, Versluijs MM, et al. Choosing a hospital for surgery: the importance of information on quality of care. Med Decis Making 2010;30:544-55

18. Brown PH, Theoharides $\mathrm{C}$. Health-seeking behavior and hospital choice in China's New Cooperative Medical System. Health Econ 2009;18(Suppl 2):S47-64.

19. Qian D, Lucas H, Chen J, et al. Determinants of the use of different types of health care provider in urban China: a tracer illness study of URTI. Health Policy 2010;98:227-35.

20. Center for Health Statistics and Information, the People's Republic of China. An analysis report of national health service survey in China, 2013. Beijing: Pecking Union Medical College Press, 2014.

21. Wang Q, Zhang D, Hou Z. Insurance coverage and socioeconomic differences in patient choice between private and public health care providers in China. Soc Sci Med 2016;170:124-32.

22. Hu X, Cook S, Salazar MA. Internal migration and health in China Lancet 2008;372:1717-9.

23. Qiu P, Yang Y, Zhang J, et al. Rural-to-urban migration and its implication for new cooperative medical scheme coverage and utilization in China. BMC Public Health 2011;11:520.

24. Ministry of Human Resources and Social Security, the People's Republic of China. Interim measures for the transferability of basic medical insurance account among internal migrants. 2009-12-31 http://www.mohrss.gov.cn/gkml/xxgk/201407/t20140717_136139. html

25. Meng Q, Fang $\mathrm{H}$, Liu X, et al. Consolidating the social health insurance schemes in China: towards an equitable and efficient health system. Lancet 2015;386:1484-92.

26. National Health and Family Planning Commission of China. 2015 Report on China's migrant population development. Beijing: China Population Publishing House, 2015.

27. National Health and Family Planning Commission of China. China health statistical yearbook 2016. Beijing: Peking Union Medical College Press, 2017.

28. Yip WC, Wang H, Liu Y. Determinants of patient choice of medical provider: a case study in rural China. Health Policy Plan 1998;13:311-22.

29. Jin Y, Hou Z, Zhang D. Determinants of health insurance coverage among people aged 45 and over in China: who buys public, private and multiple insurance. PLoS One 2016;11:e161774.

30. Information platform of Chinese urban and rural resident basic medical insurances. http://www.xnh.org.cn

31. Peng $\mathrm{Y}$, Chang $\mathrm{W}$, Zhou $\mathrm{H}$, et al. Factors associated with healthseeking behavior among migrant workers in Beijing, China. BMC Health Serv Res 2010;10:69.

32. Yin $H$, Lan $Y$, Ying L. Study on the division and cooperation among medical institutions based on hierarchical diagnosis and treatment system. Chinese Hospitals 2014:1-3. [In Chinese].

33. Yip W, Hsiao WC. What drove the cycles of Chinese health system reforms? Health Systems \& Reform 2015;1:52-61.

34. Qian $Y$, Hou Z, Wang W, et al. Integrated care reform in urban China: a qualitative study on design, supporting environment and implementation. Int J Equity Health 2017;16:185.

35. Yang $H$, Huang $X$, Zhou Z, et al. Determinants of initial utilization of community healthcare services among patients with major non-communicable chronic diseases in South China. PLoS One 2014;9:e116051.

36. Flatø $\mathrm{H}$, Zhang $\mathrm{H}$. Inequity in level of healthcare utilization before and after universal health coverage reforms in China: evidence from household surveys in Sichuan Province. Int J Equity Health 2016;15:96

37. Song X, Zou G, Chen W, et al. Health service utilisation of ruralto-urban migrants in Guangzhou, China: does employment status matter? Trop Med Int Health 2017;22:82-91.

38. Nielsen MB, Knardahl S. The healthy worker effect: do health problems predict participation rates in, and the results of, a follow-up survey? Int Arch Occup Environ Health 2016;89:231-8.

39. Si-zhang H, Da-ren Z, Bo-xing S, et al. Practical situation and consideration of classified diagnosis and treatment in China. Modern Hospital Management 2015:20-2. [In Chinese].

40. Wei X, Pearson S, Zhang Z, et al. Comparing knowledge and use of health services of migrants from rural and urban areas in Kunming City, China. J Biosoc Sci 2010;42:743-56. 
41. Lam KK, Johnston JM. Health insurance and healthcare utilisation for Shenzhen residents: a tale of registrants and migrants? BMC Public Health 2012;12:868.

42. Zou G, Zeng Z, Chen W, et al. Self-reported illnesses and service utilisation among migrants working in small-to medium sized enterprises in Guangdong, China. Public Health 2015;129:970-8.
43. Zhang L, Liu S, Zhang G, et al. Internal migration and the health of the returned population: a nationally representative study of China. BMC Public Health 2015;15:719.

44. State Council. State Council's opinon on the integration of urban and rural social health insurance schemes. 2016-1-12 http://www.gov.cn/ zhengce/content/2016-01/12/content_10582.htm 